\title{
ON A SIMILARITY SOLUTION OF MHD BOUNDARY LAYER FLOW OVER A MOVING VERTICAL CYLINDER
}

\author{
MARYEM AMKADNI AND ADNANE AZZOUZI
}

Received 22 March 2006; Revised 2 July 2006; Accepted 26 July 2006

The steady flow of an incompressible electrically conducting fluid over a semi-infinite moving vertical cylinder in the presence of a uniform transverse magnetic field is analyzed. The partial differential equations governing the flow are reduced to an ordinary differential equation, using the self-similarity transformation. The analysis deals with the existence of an exact solution to the boundary value problem by a shooting method.

Copyright (C) 2006 M. Amkadni and A. Azzouzi. This is an open access article distributed under the Creative Commons Attribution License, which permits unrestricted use, distribution, and reproduction in any medium, provided the original work is properly cited.

\section{Introduction}

The study of laminar flow over a continuously moving surface in a viscous incompressible fluid is of considerable interest in many industrial applications and a large number of papers investigating different aspects of this problem have been published. Boundary layer flow behavior on a cylinder moving in a Newtonian fluid was initially studied by Sakiadis [19], and obtained a numerical solution using a similarity transformation. Later, this problem has received the attention of certain researchers (see $[9,14,18])$.

More recently the problem of MHD flow over infinite surfaces has become more important due to the possibility of applications in areas like nuclear fusion, chemical engineering, medicine, and high-speed, noiseless printing. Problem of MHD flow in the vicinity of infinite plate has been studied intensively by a number of investigators (see, e.g., $[12,16,17,20-23]$ and the references therein). But only very few authors studied the flow past semi-infinite vertical cylinder (see, e.g., $[1,2,5,10,13]$ and the references therein). It may be remarked that most exact solutions in fluid mechanics and MHD are similarity solutions in the sense that the number of independent variable is reduced by one or more.

Most of previous investigations were concerned with numerical studies and there are only few papers in the literature that deal with a theoretical analysis of problem of MHD flow along a vertical cylinder, however, an important number of theoretical investigations

Hindawi Publishing Corporation

Differential Equations and Nonlinear Mechanics

Volume 2006, Article ID 52765, Pages 1-9

DOI 10.1155/DENM/2006/52765 
are concerned with flow past vertical and flat plates without magnetic field (see, e.g., $[3,4,7,8,11,15]$ and the references therein). The subject of the present note is to give an analytic investigation to the problem of boundary layer in a laminar flow of a viscous incompressible and electrically conducting fluid past a permeable moving vertical semi-infinite cylinder under the action of a uniform magnetic field in the case of a linear external velocity. The governing boundary layer equations with initial and boundary conditions are reduced to an ordinary differential equation which is solved using a shooting method, and favorable conditions for the existence of solutions are established.

\section{Mathematical formulation}

We consider a steady laminar and incompressible viscous MHD flow past a moving permeable semi-infinite vertical cylinder of radius $R$. The applied transverse magnetic field $B_{0}$ is assumed to be uniform. All fluid properties are assumed to be constant and the magnetic Reynolds number is assumed to be small so that the magnetic field can be neglected. No electric field is assumed to exist. Axial coordinate $x$ is measured along the axis of the cylinder. The radial coordinate $r$ is measured normal to the axis of cylinder. We denote by $u_{e}(x)=u_{\infty} x$ the external velocity with $u_{\infty}>0$. Under these assumptions and with the boundary layer approximation, the governing equations describing the problem are

$$
\begin{gathered}
\frac{\partial(r u)}{\partial x}+\frac{\partial(r v)}{\partial r}=0 \\
u \frac{\partial u}{\partial x}+v \frac{\partial u}{\partial x}=\frac{\nu}{r} \frac{\partial}{\partial r}\left(r \frac{\partial u}{\partial r}\right)+u_{e} \frac{d u_{e}}{d x}+\frac{\sigma B^{2}}{\rho}\left(u_{e}-u\right),
\end{gathered}
$$

with initial and boundary conditions

$$
u(R, x)=u_{w} x, \quad v(R, x)=-v_{w}, \quad u(\infty, x)=u_{e}(x),
$$

we denote by $u$ and $v$ the velocity components in the $x$ and $r$ directions, respectively. $\nu$ is the kinematic viscosity, $\rho$ is the fluid density, and $\sigma$ is the electric conductivity of the fluid. $v_{\omega}$ is the suction/injection parameter, with $v_{\omega}>0$ corresponding to the wall suction, $v_{\omega}<0$ corresponding to the wall blowing, and the case $v_{\omega}=0$ characterizing the impermeable wall. In our following analysis we assume that $v_{\omega}>0$ and $u_{\omega}>0$.

The stream function $\psi$ is defined by $r u=\partial \psi / \partial r$ and $r v=-\partial \psi / \partial x$, substituting these expressions in (2.1)-(2.2), the continuity equation is automatically satisfied and we obtain the boundary value problem

$$
\begin{gathered}
\frac{1}{r^{2}} \frac{\partial \psi}{\partial r} \frac{\partial^{2} \psi}{\partial x \partial r}+\frac{1}{r^{3}} \frac{\partial \psi}{\partial x} \frac{\partial \psi}{\partial r}-\frac{1}{r^{2}} \frac{\partial \psi}{\partial x} \frac{\partial^{2} \psi}{\partial r^{2}} \\
=\frac{\nu}{r^{3}} \frac{\partial \psi}{\partial r}-\frac{\nu}{r^{2}} \frac{\partial^{2} \psi}{\partial r^{2}}+\frac{\nu}{r} \frac{\partial^{3} \psi}{\partial r^{3}}+u_{e} \frac{d u_{e}}{d x}+\frac{\sigma B_{0}^{2}}{\rho}\left(u_{e}-\frac{1}{r} \frac{\partial \psi}{\partial r}\right), \\
\frac{\partial \psi}{\partial r}(R, x)=R u_{\omega} x, \quad \frac{\partial \psi}{\partial x}(R, x)=R v_{\omega}, \quad \lim _{r \rightarrow \infty}\left(\frac{1}{r} \frac{\partial \psi}{\partial r}(r, x)\right)=u_{\infty} x .
\end{gathered}
$$


We look for self-similar solutions under the form

$$
\psi(r, x)=\sqrt{\frac{\nu u_{\infty} R}{2}} x f(t),
$$

where $f$ is the dimensionless stream function and $t=\sqrt{u_{\infty} R / 2 \nu}\left(\left(r^{2}-R^{2}\right) / R\right)$ is the similarity variable.

In terms of this variable, the governing equation and boundary conditions (2.3) are transformed into

$$
\begin{gathered}
(K t+2 R) f^{\prime \prime \prime}(t)+K f^{\prime \prime}(t)+f(t) f^{\prime \prime}(t)-f^{\prime 2}(t)-M\left(f^{\prime}(t)-1\right)+1=0, \\
f(0)=a, \quad f^{\prime}(0)=b, \quad f^{\prime}(\infty)=1,
\end{gathered}
$$

with

$$
K=2 \sqrt{\frac{2 v}{u_{\infty} R}}, \quad a=\frac{v_{\omega} R}{\sqrt{\nu u_{\infty} R}}, \quad b=\frac{u_{\omega}}{2 u_{\infty}}, \quad M=\frac{\sigma B_{0}^{2}}{\rho u_{\infty}} .
$$

Note that $M>0$ is the magnetic parameter and $a>0$ plays the role of suction parameter.

\section{Main result}

The objective in this section is to establish a sufficient condition for the existence of exact solutions of the problem (2.5)-(2.6) with respect to the three parameters $a, b$, and $M$. For this we will study the related initial value problem

$$
\begin{gathered}
(K t+2 R) f^{\prime \prime \prime}(t)+K f^{\prime \prime}(t)+f(t) f^{\prime \prime}(t)-f^{\prime 2}(t)-M\left(f^{\prime}(t)-1\right)+1=0, \\
f(0)=a, \quad f^{\prime}(0)=b, \quad f^{\prime \prime}(0)=c,
\end{gathered}
$$

where the real $c$ is the shooting parameter. Problem (3.1) has a unique local solution $f_{c}$ defined on its maximal interval of existence $\left[0, T_{c}\right), T_{c} \leq \infty$. This solution is of class $C^{\infty}$ on $\left[0, T_{c}\right)$. Let us note that if $T_{c}<\infty$, then

$$
\lim _{t \rightarrow T_{c}}\left|f_{c}(t)\right|+\left|f_{c}^{\prime}(t)\right|+\left|f_{c}^{\prime \prime}(t)\right|=+\infty
$$

Theorem 3.1. For $a>0$ and $b>(3 / 2)\left(\sqrt{M^{2} / 4+(4 / 3)(M+1)}-M / 2\right)$, if

$$
-\sqrt{\frac{1}{R}\left(\frac{b^{3}}{3}+\frac{M}{2} b^{2}-(M+1) b\right)}<c<0,
$$

the problem (2.5)-(2.6) has at most one solution.

To prove this theorem, we use the following lemmas.

Lemma 3.2. If a solution $f_{c}$ of problem (3.1) is defined on $\left[0, T_{c}\right)$ with $T_{c}<\infty$, then $f_{c}, f_{c}^{\prime}$, and $f_{c}^{\prime \prime}$ are unbounded for $t \rightarrow T_{c}$. 
4 A similarity solution of MHD boundary layer flow

Proof. We use the same idea in [6] for the Falkner-skan equation. If $f_{c}^{\prime \prime}$ were bounded for $t \rightarrow T_{c}$ then $f_{c}^{\prime}$ and $f_{c}$ would also be bounded. But we have

$$
\lim _{t \rightarrow T_{c}}\left|f_{c}(t)\right|+\left|f_{c}^{\prime}(t)\right|+\left|f_{c}^{\prime \prime}(t)\right|=+\infty
$$

a contradiction. If $f_{c}^{\prime}$ were bounded for $t \rightarrow T_{c}, f_{c}$ would also be bounded, on the other hand integrating $(3.1)_{1}$ between the limits 0 and $t$ we get

$$
K t f_{c}^{\prime \prime}(t)+2 R\left(f_{c}^{\prime \prime}(t)-c\right)=-f_{c}(t) f_{c}^{\prime}(t)+a b+2 \int_{0}^{t} f_{c}^{\prime 2}(s) d s+M\left(f_{c}(t)-a\right)-(M+1) t
$$

this implies that $f_{c}^{\prime \prime}$ is bounded and we have seen that this is impossible.

Now suppose $f_{c}$ were bounded for $t \rightarrow T_{c}$, by integration of (3.5) between 0 and $t$ we obtain

$$
\begin{aligned}
(K t+ & 2 R) f_{c}^{\prime}(t)+\frac{1}{2} f_{c}^{2}(t)-K f_{c}(t)-(2 c R+a b-M a) t+(M+1) \frac{t^{2}}{2}-M \int_{0}^{t} f_{c}(s) d s \\
& =2 \int_{0}^{t} \int_{0}^{\eta} f_{c}^{\prime 2}(\eta) d \eta d t,
\end{aligned}
$$

since $f_{c}^{\prime}$ is unbounded for $t \rightarrow T_{c}$, then $\int_{0}^{t} \int_{0}^{\eta} f_{c}^{\prime 2}(\eta) d \eta d t$ is also unbounded. Whereas $\int_{0}^{t} \int_{0}^{\eta} f_{c}^{\prime 2}(\eta) d \eta d t$ is a monotonic function of $t$, therefore it tends to infinity and also does $f_{c}^{\prime}(t)$.

If we put $\omega=\int_{0}^{t} \int_{0}^{\eta} f_{c}^{\prime 2}(\eta) d \eta d t$, then

$$
\omega^{\prime \prime}=f_{c}^{\prime 2} \sim \frac{4}{\left(K T_{c}+2 R\right)^{2}} \omega^{2} \quad \text { for } t \longrightarrow T_{c} .
$$

Multiplying by $\omega^{\prime}$, integrating and using the fact that $\omega$ tends to infinity for $t \rightarrow T_{c}$, we obtain

$$
\begin{aligned}
& \frac{1}{2} \omega^{\prime 2} \sim \frac{4}{3\left(K T_{c}+2 R\right)^{2}} \omega^{3}, \\
& \omega^{\prime} \sim \frac{\sqrt{8}}{\sqrt{3}\left(K T_{c}+2 R\right)} \omega^{3 / 2} .
\end{aligned}
$$

By the theory of indeterminate forms it follows that

$$
\omega^{-1 / 2} \sim c_{1}\left(T_{c}-t\right) \quad \text { for } t \longrightarrow T_{c},
$$

where $c_{1}$ is a positive constant. Hence

$$
f_{c}^{\prime} \sim \frac{2}{K T_{c}+2 R} \omega \sim c_{2}\left(T_{c}-t\right)^{-2}
$$


where $c_{2} \neq 0$. Integrating, we get

$$
f_{c} \sim c_{2}\left(T_{c}-t\right)^{-1}
$$

this contradicts the fact that $f_{c}$ is bounded for $t \rightarrow T_{c}$.

Lemma 3.3. Let $a>0, b>(3 / 2)\left(\sqrt{M^{2} / 4+(4 / 3)(M+1)}-M / 2\right)$, and

$$
-\sqrt{\left(\frac{1}{R}\right)\left(\frac{b^{3}}{3}+\left(\frac{M}{2}\right) b^{2}-(M+1) b\right)}<c<0,
$$

then $f_{c}^{\prime}>1$ and $f_{c}>0$ on $\left[0, T_{c}\right)$.

Proof. Since $f_{c}^{\prime}(0)=b>1$, then $f_{c}^{\prime}>1$ on some $\left[0, t_{0}\right), 0<t_{0}<T_{c}$. Since $f_{c}(0)=a>0$, we obtain that $f_{c}>0$ on $\left[0, t_{0}\right)$. Suppose that there exists $t_{0} \leq t_{1}<T_{c}$ such that $f_{c}^{\prime}\left(t_{1}\right)=1$ and $f_{c}^{\prime}>1$ on $\left[0, t_{1}\right)$. We introduce the function $E$ defined by

$$
\begin{aligned}
E(t)= & \left(\frac{K t}{2}+R\right) f_{c}^{\prime \prime 2}(t)-\frac{f_{c}^{\prime 3}(t)}{3}-\frac{M}{2} f_{c}^{\prime 2}(t)+(M+1) f_{c}^{\prime}(t) \\
& +\frac{K}{2} \int_{0}^{t} f_{c}^{\prime \prime 2}(s) d s \quad \forall t \in\left[0, T_{c}\right)
\end{aligned}
$$

then by $(3.1)_{1}$ we get

$$
E^{\prime}(t)=-f_{c}(t) f_{c}^{\prime \prime 2}(t) \leq 0 \quad \text { on }\left[0, t_{1}\right)
$$

In addition we have $E(0)=R c^{2}-b^{3} / 3-(M / 2) b^{2}+(M+1) b \leq 0$, it follows that $E\left(t_{1}\right)=$ $E(0)=0$. Thus $E(t)=E^{\prime}(t)=0$ for all $t \in\left[0, t_{1}\right)$. Consequently $f_{c}^{\prime \prime}(t)=0$ for all $t \in\left[0, t_{1}\right)$, which implies $c=0$; a contradiction. Hence $f_{c}^{\prime}>1$ and so $f_{c}>0$.

Lemma 3.4. Let $-\sqrt{(1 / R)\left(b^{3} / 3+(M / 2) b^{2}-(M+1) b\right)}<c<0$ such that $f_{c}^{\prime}$ is bounded, then $f_{c}^{\prime \prime}<0$ on $\left[0, T_{c}\right)$.

Proof. Since $f_{c}^{\prime \prime}(0)<0$ then $f_{c}^{\prime \prime}$ is negative on a neighborhood of 0 . Suppose that there exists a number $t_{0}>0$ such that $f_{c}^{\prime \prime}\left(t_{0}\right)=0$ and $f_{c}^{\prime \prime}<0$ on $\left(0, t_{0}\right)$. We will show that $f_{c}^{\prime \prime \prime}>$ 0 for $t \geq t_{0}$. In fact, because $f_{c}^{\prime \prime}<0$ on $\left(0, t_{0}\right)$ we have $f_{c}^{\prime \prime \prime}\left(t_{0}\right) \geq 0$. Suppose that $f_{c}^{\prime \prime \prime}\left(t_{0}\right)=$ 0 , then it follows from (2.5) that

$$
\left(1-f_{c}^{\prime}\left(t_{0}\right)\right)\left(1+f_{c}^{\prime}\left(t_{0}\right)+M\right)=0
$$

which implies that $f_{c}^{\prime}\left(t_{0}\right)=1$ or $f_{c}^{\prime}\left(t_{0}\right)=-(1+M)$, this contradicts the fact that $f_{c}^{\prime}>$ 1 and then $f_{c}^{\prime \prime \prime}\left(t_{0}\right)>0$. Now we suppose that there exists a number $t_{1}>t_{0}$ such that $f^{\prime \prime \prime}\left(t_{1}\right)=0$, let us observe that $f_{c}^{(4)}\left(t_{1}\right) \leq 0$ and $f_{c}^{\prime \prime}\left(t_{1}\right) \geq 0$. Differentiation of (2.5) yields

$$
(K t+2 R) f_{c}^{(4)}(t)+2 K f_{c}^{\prime \prime \prime}(t)+f_{c}(t) f_{c}^{\prime \prime \prime}(t)-f_{c}^{\prime}(t) f_{c}^{\prime \prime}(t)-M f_{c}^{\prime \prime}(t)=0,
$$


6 A similarity solution of MHD boundary layer flow

and hence at $t=t_{1}$, we obtain

$$
\left(K t_{1}+2 R\right) f_{c}^{(4)}\left(t_{1}\right)-M f_{c}^{\prime \prime}\left(t_{1}\right)=f_{c}^{\prime}\left(t_{1}\right) f_{c}^{\prime \prime}\left(t_{1}\right)
$$

thus we deduce that $f_{c}^{\prime}\left(t_{1}\right) \leq 0$; a contradiction. Consequently $f_{c}^{\prime \prime \prime}>0$ and then $f_{c}^{\prime \prime}>0$ on $\left(t_{0}, \infty\right)$, we get that $f_{c}^{\prime}$ is increasing and becomes unbounded as $t$ tends to infinity, this contradicts the fact that $f_{c}^{\prime}$ is bounded and then $f_{c}^{\prime \prime}<0$ on $\left[0, T_{c}\right)$.

Proof of Theorem 3.1. First let us distinguish two cases.

(1) For any $-\sqrt{(1 / R)\left(b^{3} / 3+(M / 2) b^{2}-(M+1) b\right)}<c<0$, $f_{c}^{\prime}$ is unbounded.

(2) There exists a real $c_{1}$ satisfying $-\sqrt{(1 / R)\left(b^{3} / 3+(M / 2) b^{2}-(M+1) b\right)}<c_{1}<0$ such that $f_{c_{1}}^{\prime}$ is bounded.

In the case (1) it is clear that $f_{c}^{\prime}$ is not a solution of (2.5)-(2.6) since the boundary condition at infinity could not be satisfied. Then to investigate solutions of problem (2.5)-(2.6), it remains to consider the case (2). Since $f_{c_{1}}^{\prime}$ is bounded, then from function $E$ we have $f_{c_{1}}^{\prime \prime}$ is also bounded on $\left[0, T_{c_{1}}\right)$. Assume that $T_{c_{1}}$ is finite, since $f_{c_{1}}^{\prime}$ is bounded, then $f_{c_{1}}$ is also bounded. But this contradicts Lemma 3.2, consequently $T_{c_{1}}=+\infty$. Hence from Lemma 3.3, there exists $L \in(0,+\infty]$ such that $\lim _{t \rightarrow+\infty} f_{c_{1}}(t)=L$, assume that $L<+\infty$, this implies in particular the existence of a sequence $\left(t_{n}\right)$ tending to $+\infty$ with $n$ such that $\lim _{n \rightarrow+\infty} f_{c_{1}}^{\prime}\left(t_{n}\right)=0$, by using the function $E$, we get $0 \leq E(+\infty) \leq E(0) \leq 0$ and then $E(t)=E^{\prime}(t)=0$ for all $t \in[0,+\infty)$. Hence $f_{c_{1}}^{\prime \prime}(t)=0$ for all $t \in[0,+\infty)$, which implies $c=0$, this is impossible. We use again the function $E$ to find the limit of $f_{c_{1}}^{\prime \prime}$, since $E(t) \leq E(0)$ we have

$$
\left(\frac{K t}{2}+R\right) f_{c_{1}}^{\prime \prime 2}+\frac{K}{2} \int_{0}^{t} f_{c_{1}}^{\prime \prime 2}(s) d s \leq B
$$

where $B$ is a constant. There exists $t_{2} \in(0, t)$ such that

$$
R f_{c_{1}}^{\prime \prime 2}(t)+\frac{K t}{2} f_{c_{1}}^{\prime \prime 2}(t)+\frac{K}{2} f_{c_{1}}^{\prime \prime 2}\left(t_{2}\right) t \leq B
$$

then

$$
f_{c_{1}}^{\prime \prime 2}(t) \leq \frac{1}{t}\left(\frac{2 B}{K}\right)
$$

thus we get $\lim _{t \rightarrow+\infty} f_{c_{1}}^{\prime \prime}(t)=0$. Finally we prove that $f_{c_{1}}^{\prime}$ tends to 1 as $t$ approaches infinity, for this suppose that $f_{c_{1}}^{\prime}$ is oscillating indefinitely, this implies the existence of two sequences:

$\left(t_{n}\right)_{n}$ the sequence of points where the local maximums of $f_{c_{1}}^{\prime}$ are reached, $\left(\tau_{n}\right)_{n}$ the sequence of points where the local minimums of $f_{c_{1}}^{\prime}$ are reached,

then $\left(t_{n}\right)_{n}$ and $\left(\tau_{n}\right)_{n}$ are tending to $+\infty$ with $n$, and satisfying

$$
f_{c_{1}}^{\prime \prime}\left(t_{n}\right)=f_{c_{1}}^{\prime \prime}\left(\tau_{n}\right)=0, \quad f_{c_{1}}^{\prime \prime \prime}\left(t_{n}\right)<0, \quad f_{c_{1}}^{\prime \prime \prime}\left(\tau_{n}\right)>0 \quad \forall n \in \mathbb{N} .
$$


By using the polynomial $p(x)=x^{2}+M x-(M+1), x \in \mathbb{R}^{+}$, and $(3.1)_{1}$ we get

$$
\begin{aligned}
\left(K t_{n}+2 R\right) f_{c_{1}}^{\prime \prime \prime}\left(t_{n}\right) & =f_{c_{1}}^{\prime 2}\left(t_{n}\right)+M f_{c_{1}}^{\prime}\left(t_{n}\right)-(M+1)=p\left(f_{c_{1}}^{\prime}\left(t_{n}\right)\right)<0, \\
\left(K \tau_{n}+2 R\right) f_{c_{1}}^{\prime \prime \prime}\left(\tau_{n}\right) & =f_{c_{1}}^{\prime 2}\left(\tau_{n}\right)+M f_{c_{1}}^{\prime}\left(\tau_{n}\right)-(M+1)=p\left(f_{c_{1}}^{\prime}\left(\tau_{n}\right)\right)>0,
\end{aligned}
$$

and we deduce that $0<f_{c_{1}}^{\prime}\left(t_{n}\right)<1$ and $f_{\mathcal{c}_{1}}^{\prime}\left(\tau_{n}\right)>1 \forall n \in \mathbb{N}$; a contradiction.

Then it follows that $f_{c_{1}}^{\prime}$ is monotone on $\left(t_{1},+\infty\right)$, where $t_{1}$ is large enough. Since $f_{c_{1}}^{\prime}$ is bounded, hence there exists $l \in \mathbb{R}^{+}$such that

$$
\lim _{t \rightarrow+\infty} f_{c_{1}}^{\prime}(t)=l
$$

then

$$
f_{c_{1}} \sim l t \text { for } t \longrightarrow+\infty
$$

and from identity (3.5) we have

$$
(K t+2 R) f_{c_{1}}^{\prime \prime} \sim l^{2} t+M l t-(M+1) t \quad \text { for } t \longrightarrow+\infty .
$$

We deduce that for $t \rightarrow+\infty$

$$
\begin{aligned}
f_{\mathcal{C}_{1}}^{\prime \prime} & \sim \frac{1}{K t}\left[l^{2} t+M l t-(M+1) t\right] \\
& \sim \frac{1}{K} p(l) .
\end{aligned}
$$

Thus, since $\lim _{t \rightarrow+\infty} f_{c_{1}}^{\prime \prime}(t)=0$, we get $p(l)=0$ and then $l=1$. Thus $f_{c_{1}}$ is a solution of (2.5)-(2.6).

Suppose that there exists another real $c_{2}$ satisfying $-\sqrt{(1 / R)\left(b^{3} / 3+(M / 2) b^{2}-(M+1) b\right)}$ $<c_{2}<0$ such that $f_{c_{2}}^{\prime}$ is bounded, then $f_{c_{2}}$ is also a solution of (2.5)-(2.6).

Assume that $c_{1}>c_{2}$ and consider the function $g=f_{c_{1}}-f_{c_{2}}$, we have $g^{\prime}(0)=g^{\prime}(\infty)=0$ and $g^{\prime \prime}(0)>0$, then $g^{\prime}$ is a positive maximum at some point $t_{0}>0$ such that $g^{\prime}>0$ on $\left(0, t_{0}\right]$, therefore we have

$$
g\left(t_{0}\right)>0, \quad g^{\prime}\left(t_{0}\right)>0, \quad g^{\prime \prime}\left(t_{0}\right)=0, \quad g^{\prime \prime \prime}\left(t_{0}\right) \leq 0
$$

From (2.5) we obtain

$$
\begin{aligned}
& \left(K t_{0}+2 R\right) f_{c_{1}}^{\prime \prime \prime}\left(t_{0}\right)+K f_{c_{1}}^{\prime \prime}\left(t_{0}\right)+f_{c_{1}}\left(t_{0}\right) f_{c_{1}}^{\prime \prime}\left(t_{0}\right)+1-f_{c_{1}}^{\prime}\left(t_{0}\right)^{2}+M\left(1-f_{c_{1}}^{\prime}\left(t_{0}\right)\right)=0, \\
& \left(K t_{0}+2 R\right) f_{c_{2}}^{\prime \prime \prime}\left(t_{0}\right)+K f_{c_{2}}^{\prime \prime}\left(t_{0}\right)+f_{c_{2}}\left(t_{0}\right) f_{c_{2}}^{\prime \prime}\left(t_{0}\right)+1-f_{c_{2}}^{\prime}\left(t_{0}\right)^{2}+M\left(1-f_{c_{2}}^{\prime}\left(t_{0}\right)\right)=0 .
\end{aligned}
$$

Using (3.29) we obtain

$$
\left(K t_{0}+2 R\right) g^{\prime \prime \prime}\left(t_{0}\right)=-f_{c_{2}}^{\prime \prime}\left(t_{0}\right) g\left(t_{0}\right)+g^{\prime}\left(t_{0}\right)\left(f_{c_{2}}^{\prime}\left(t_{0}\right)+f_{c_{1}}^{\prime}\left(t_{0}\right)+M\right) .
$$


From Lemma 3.4 we have $f_{\mathcal{c}_{2}}^{\prime \prime}\left(t_{0}\right)<0$ and then the right-hand side is positive but $g^{\prime \prime \prime}\left(t_{0}\right) \leq$ 0 ; a contradiction. Therefore $c_{1}=c_{2}$ and we conclude that if there exists a real $c$ in the interval $]-\sqrt{(1 / R)\left(b^{3} / 3+(M / 2) b^{2}-(M+1) b\right)}, 0$ [ such that $f_{c}$ is a solution of $(2.5)-(2.6)$, then $c$ is unique. This ends the proof of Theorem 3.1.

\section{References}

[1] E. M. Abo-Eldahab and A. M. Salem, MHD Flow and heat transfer of non-Newtonian powerlaw fluid with diffusion and chemical reaction on a moving cylinder, Heat and Mass Transfer 41 (2005), no. 8, 703-708.

[2] K. L. Arora and P. R. Gupta, Magnetohydrodynamic flow between two rotating coaxial cylinders under radial magnetic field, Physics of Fluids 15 (1972), no. 6, 1146-1148.

[3] W. H. H. Banks, Similarity solutions of the boundary-layer equations for a stretching wall, Journal de Mécanique Théorique et Appliquée 2 (1983), no. 3, 375-392.

[4] Z. Belhachmi, B. Brighi, and K. Taous, On a family of differential equations for boundary layer approximations in porous media, European Journal of Applied Mathematics 12 (2001), no. 4, 513-528.

[5] T. S. Chen and C. F. Yuh, Combined heat and mass transfer in natural convection along a vertical cylinder, International Journal of Heat and Mass Transfer 23 (1980), no. 4, 451-461.

[6] W. A. Coppel, On a differential equation of boundary-layer theory, Philosophical Transactions of the Royal Society of London 253 (1960), 101-136.

[7] L. J. Crane, Flow past a stretching plate, Zeitschrift für Angewandte Mathematik und Physik 21 (1970), no. 4, 645-647.

[8] V. M. Falkner and S. W. Skan, Solutions of the boundary layer equations, Philosophical Magazine 7 (1931), no. 12, 865-896.

[9] P. Ganesan and P. Loganathan, Radiation and mass transfer effects on flow of an incompressible viscous fluid past a moving vertical cylinder, International Journal of Heat and Mass Transfer 45 (2002), no. 21, 4281-4288.

[10] _ Magnetic field effect on a moving vertical cylinder with constant heat flux, Heat and Mass Transfer 39 (2003), no. 5-6, 381-386.

[11] M. Guedda, Multiple solutions of mixed convection boundary-layer approximations in a porous medium, Applied Mathematics Letters 19 (2006), no. 1, 63-68.

[12] A. S. Gupta, Laminar free convection flow of an electrically conducting fluid from a vertical plate with uniform surface heat flux and variable wall temperature in the presence of a magnetic field, Zeitschrift für Angewandte Mathematik und Physik 13 (1962), no. 4, 324-333.

[13] M. A. Hossain and M. Ahmed, MHD forced and free convection boundary layer flow near the leading edge, International Journal of Heat and Mass Transfer 33 (1990), no. 3, 571-575.

[14] J. Krani and V. Pecho, The thermal laminar boundary layer on a continuous cylinder, International Journal of Heat and Mass Transfer 21 (1978), no. 1, 43-47.

[15] E. Magyari, I. Pop, and B. Keller, The "missing" self-similar free convection boundary-layer flow over a vertical permeable surface in a porous medium, Transport in Porous Media 46 (2002), no. 1, 91-102.

[16] I. Pop, M. Kumari, and G. Nath, Conjugate MHD flow past a flat plate, Acta Mechanica 106 (1994), no. 3-4, 215-220.

[17] I. Pop and T.-Y. Na, A note on MHD flow over a stretching permeable surface, Mechanics Research Communications 25 (1998), no. 3, 263-269.

[18] J. W. Rotte and W. J. Beek, Some models for the calculation of heat transfer coefficients to a moving continuous cylinder, Chemical Engineering Science 24 (1969), no. 4, 705-716.

[19] B. C. Sakiadis, Boundary-layer behavior on continuous solid surfaces: II. The boundary layer on a continuous flat surface, AIChE Journal 7 (1961), no. 2, 221-225. 
[20] H. S. Takhar, A. J. Chamkha, and G. Nath, Unsteady flow and heat transfer on a semi-infinite flat plate with an aligned magnetic field, International Journal of Engineering Science 37 (1999), no. 13, 1723-1736.

[21] _ Flow and mass transfer on a stretching sheet with a magnetic field and chemically reactive species, International Journal of Engineering Science 38 (2000), no. 12, 1303-1314.

[22] H. S. Takhar and G. Nath, Similarity solution of unsteady boundary layer equations with a magnetic field, Meccanica 32 (1997), no. 2, 157-163.

[23] H. S. Takhar, A. A. Raptis, and C. P. Perdikis, MHD asymmetric flow past a semi-infinite moving plate, Acta Mechanica 65 (1987), no. 1-4, 287-290.

Maryem Amkadni: Faculté de Mathématiques et d'Informatique, Université de Picardie Jules Verne, 33 rue Saint-Leu, Amiens 80039, France

E-mail address: maryem.amkadni@u-picardie.fr

Adnane Azzouzi: Faculté de Mathématiques et d'Informatique, Université de Picardie Jules Verne, 33 rue Saint-Leu, Amiens 80039, France

E-mail address: adnane.azzouzi@u-picardie.fr 


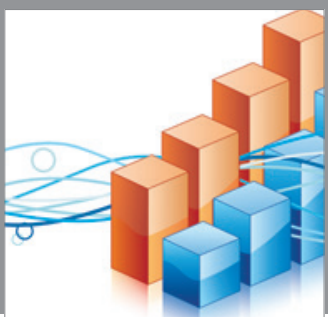

Advances in

Operations Research

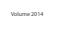

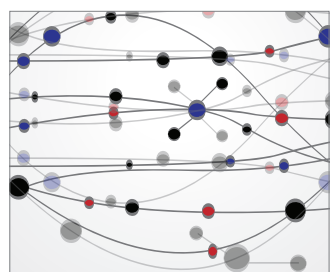

\section{The Scientific} World Journal
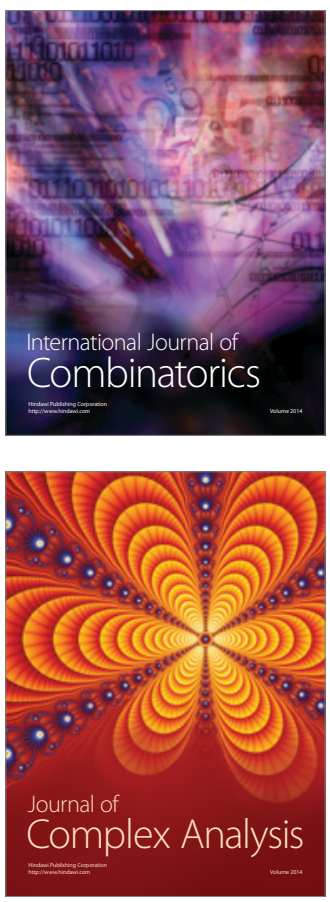

International Journal of

Mathematics and

Mathematical

Sciences
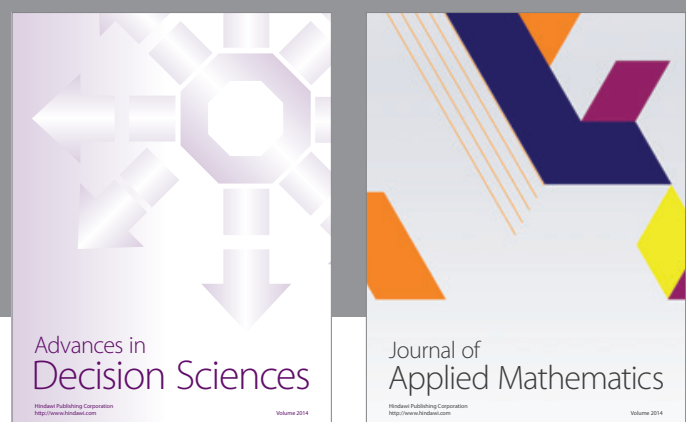

Journal of

Applied Mathematics
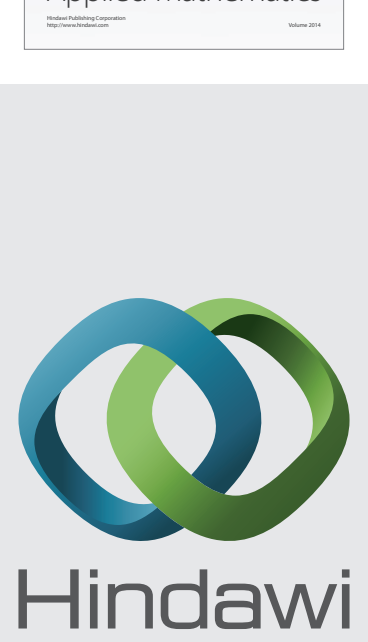

Submit your manuscripts at http://www.hindawi.com
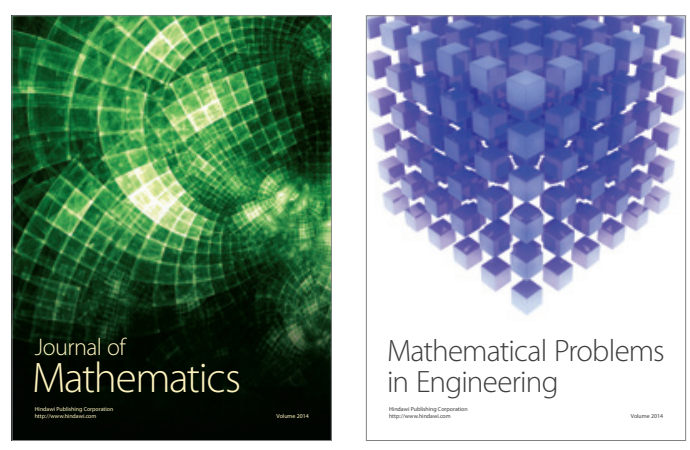

Mathematical Problems in Engineering
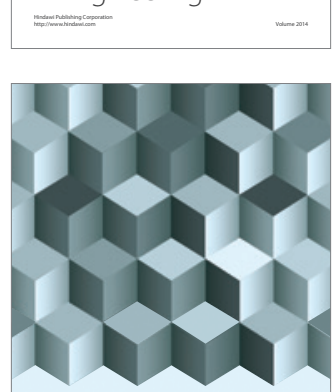

Journal of

Function Spaces
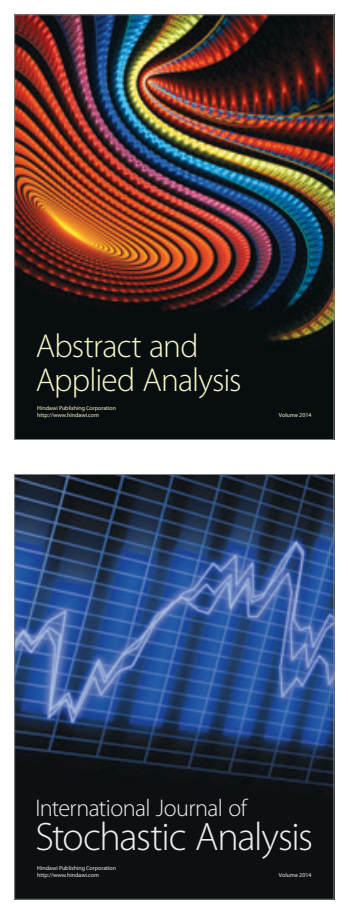

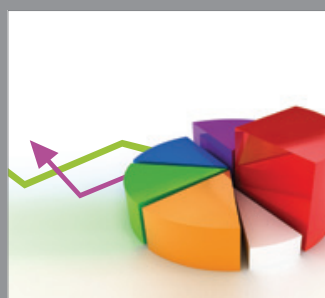

ournal of

Probability and Statistics

Promensencen
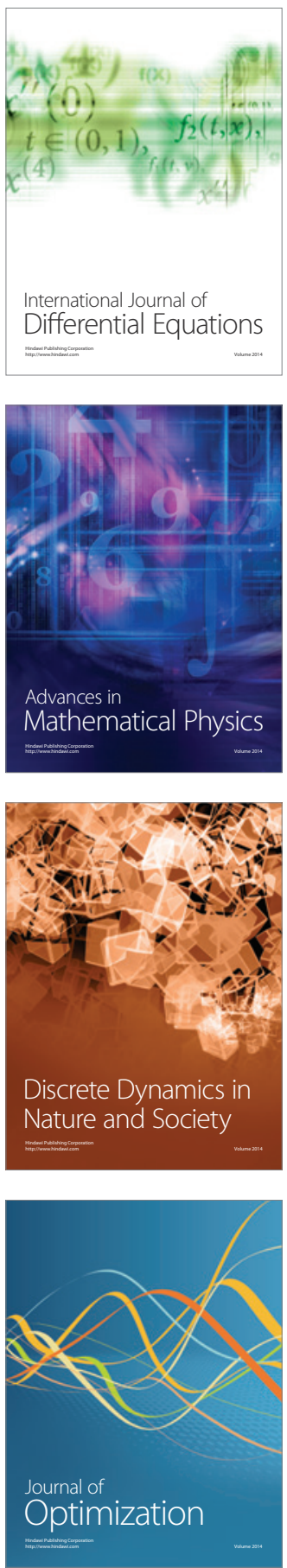